Case Report

\title{
Late Onset Ipilimumab-Induced Pericarditis and Pericardial Effusion: A Rare but Life Threatening Complication
}

\author{
Seongseok Yun, ${ }^{1}$ Nicole D. Vincelette, ${ }^{2}$ Iyad Mansour, ${ }^{1}$ Dana Hariri, ${ }^{3}$ and Sara Motamed ${ }^{4}$ \\ ${ }^{1}$ Department of Medicine, University of Arizona, Tucson, AZ 85721, USA \\ ${ }^{2}$ Molecular Pharmacology and Experimental Therapeutics, Mayo Clinic, Rochester, MN 55905, USA \\ ${ }^{3}$ Department of Pathology, University of Arizona, Tucson, AZ 85721, USA \\ ${ }^{4}$ Midwestern University, Arizona College of Osteopathic Medicine, Glendale, AZ 85308, USA
}

Correspondence should be addressed to Seongseok Yun; namaska97@gmail.com

Received 11 February 2015; Accepted 23 March 2015

Academic Editor: Francesco A. Mauri

Copyright (C) 2015 Seongseok Yun et al. This is an open access article distributed under the Creative Commons Attribution License, which permits unrestricted use, distribution, and reproduction in any medium, provided the original work is properly cited.

\begin{abstract}
Metastatic cutaneous melanoma has poor prognosis with 2-year survival rate of $10-20 \%$. Melanoma cells express various antigens including gp100, melanoma antigen recognized by T cells 1 (MART-1), and tyrosinase, which can induce immune-mediated anticancer response via $\mathrm{T}$ cell activation. Cytotoxic T-lymphocyte associated antigen-4 (CTLA-4) is an immune check point molecule that negatively regulates $\mathrm{T}$ cell activation and proliferation. Accordingly, recent phase III clinical trials demonstrated significant survival benefit with ipilimumab, a human monoclonal antibody (IgG1) that blocks the interaction of CTLA-4 with its ligands. Since the efficacy of ipilimumab depends on T cell activation, it is associated with substantial risk of immune mediated adverse reactions such as colitis, hepatitis, thyroiditis, and hypophysitis. We report the first case of late onset pericarditis and cardiac tamponade associated with ipilimumab treatment in patient with metastatic cutaneous melanoma.
\end{abstract}

\section{Case Presentation}

A 59-year-old male patient with no significant history of autoimmune disease presented to clinic with bleeding from a mole in the right forearm. Biopsy and mutation testing identified melanoma with $\mathrm{BRAF}^{\mathrm{V} 600 \mathrm{E}}$ mutation. PET/CT showed four FDG avid soft tissue nodules in the subcutaneous tissues of chest and back, abdominal mesentery, and right retroperitoneum. Excisional biopsy from right axillary lymph node was positive for melanin A staining and showed extracapsular invasion, confirming the diagnosis of stage Mlc metastatic melanoma. Therefore, patient received 4 cycles of ipilimumab $(3 \mathrm{mg} / \mathrm{kg})$ treatment every 3 weeks without significant adverse reaction except skin rash on the infusion site.

Twelve weeks after the last cycle of ipilimumab treatment, the patient presented to ED with acute onset chest pain and shortness of breath which started 1 day prior to the presentation. Vital sign showed BP $97 / 55 \mathrm{mmHg}$, HR 106 beats/min, RR 20 breaths/min, and $\mathrm{O}_{2}$ saturation $99 \%$ while breathing room air and temperature $36.9^{\circ} \mathrm{C}$. Physical examination revealed distant heart sound and $5 \mathrm{~cm}$ of jugular venous distension. Electrocardiogram showed low QRS voltage and $\mathrm{T}$ wave inversion on $\mathrm{V}_{1}-\mathrm{V}_{4}$ leads, and troponin I was negative. CT angiogram showed negative for pulmonary embolism; however, it demonstrated pericardial thickening and moderate sized pericardial effusion which are new compared to the prior study (Figures 1(a) and 1(b)). Subsequent echocardiogram showed septal bouncing and respiratory septal shift, suggesting ventricular interdependence and constrictive effusive physiology. Total $3 \mathrm{~L}$ of fluid was given for low blood pressure. Bedsides pericardiocentesis drained $130 \mathrm{~mL}$ of serosanguinous fluid and subxiphoid pericardial window was performed the next day. Biochemical study from pericardial fluid showed LDH $794 \mathrm{IU} / \mathrm{L}$, protein $4.3 \mathrm{~g} / \mathrm{dL}$, amylase $29 \mathrm{IU} / \mathrm{L}$, and glucose $99 \mathrm{mg} / \mathrm{dL}$. Fluid cytology, Gram stain, and culture were negative for neoplasm or microorganism, and adenosine deaminase PCR was also negative. WBC count was $19,600 / \mu \mathrm{L}$ with $90 \%$ of lymphocyte consistent with marked acute inflammation. Pathology from pericardial tissue demonstrated acute fibrinous pericarditis without any evidence of malignancy or microorganism (Figure 2). Additional examinations for autoimmune disease including 


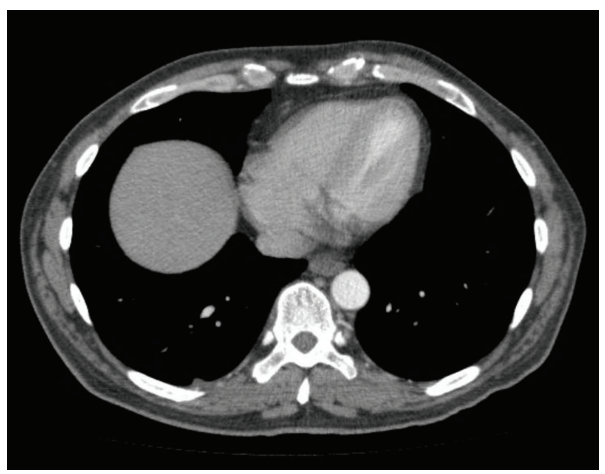

(a)

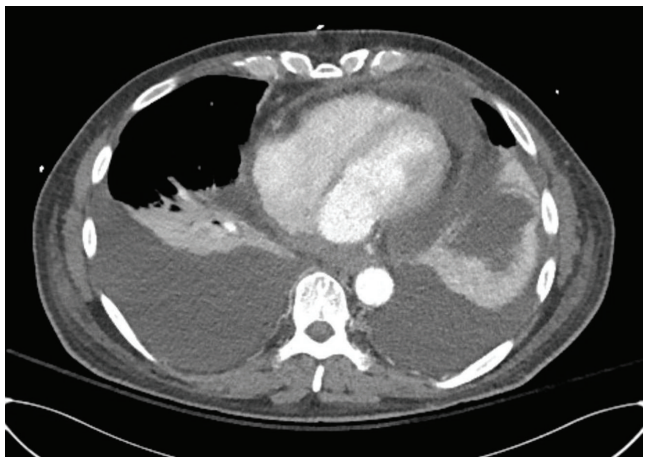

(c)

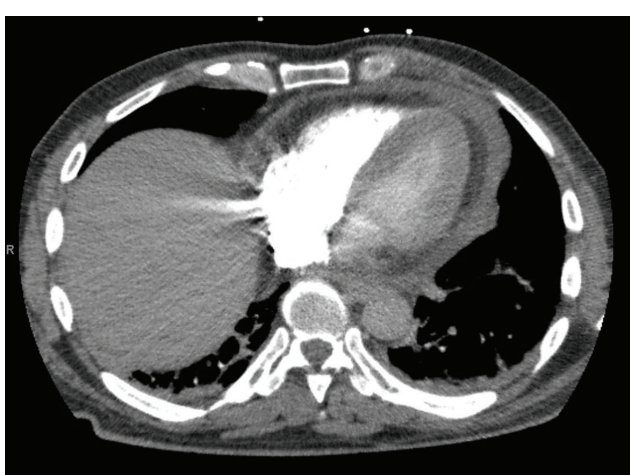

(b)

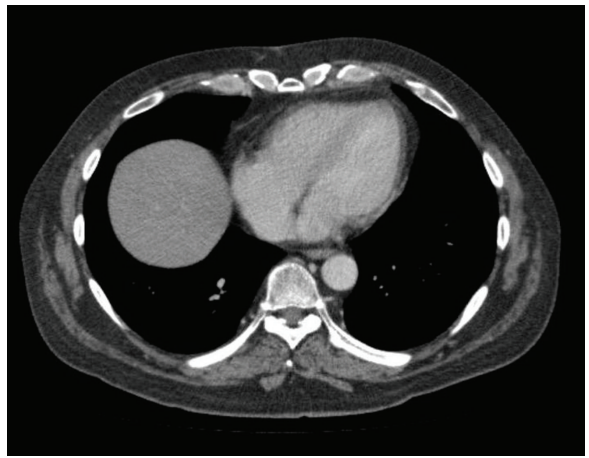

(d)

FIGURE 1: CT chest on admission showed pericardial thickening, moderate-sized pericardial effusion, and adjacent inflammatory changes within the epicardial fat and mediastinum (b), which were new compared to the prior study (a). New large bilateral pleural effusion with associated compressive atelectasis in the lower lungs and stable pericardial effusion is observed on day 10 (c), which resolved after systemic steroid treatment $(\mathrm{d})$.

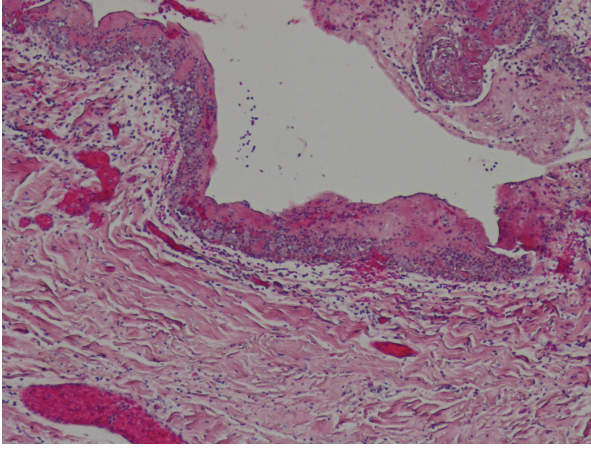

(a)

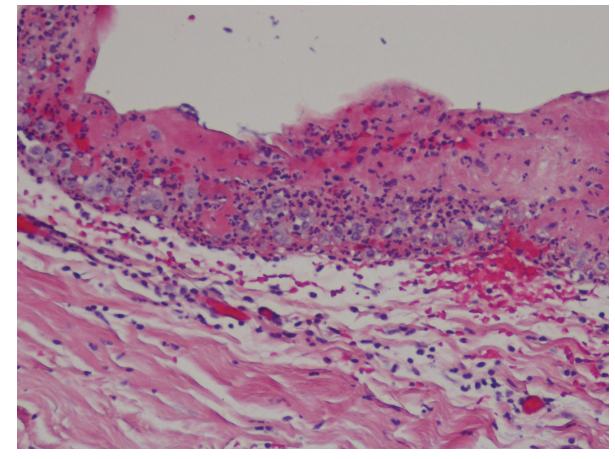

(b)

FIGURE 2: Biopsy specimen from pericardium, sections through the pericardium show acute fibrinous pericarditis, characterized by mixed inflammatory infiltrates in the pericardial wall, accompanied by abundant surface fibrin. No microorganisms were identified on hematoxylineosin-stained sections.

rheumatoid factor, anti-nuclear antibodies (ANA), double strand DNA (dsDNA), anti-neutrophil cytoplasmic antibodies (ANCA), proteinase 3, and myeloperoxidase antibodies were all negative. Further infectious work-up including blood culture, sputum culture, and respiratory viral panel were all negative as well.

Indomethacin (50 mg three times a day) was started for the treatment of acute pericarditis; however, patient developed worsening shortness of breath, generalized weakness, somnolence, and diarrhea. Blood pressure dropped down to 64/42 for which levophed and aggressive fluid resuscitation was initiated. Repeat CT scan demonstrated persistent pericardial effusion and large bilateral pleural effusion with compressive atelectasis in the lower lobes (Figure 1(c)). Thoracentesis was performed to drain $1.4 \mathrm{~L}$ of pleural fluid and biochemistry revealed borderline exudates with $\mathrm{LDH}$ ratio 0.27 , protein ratio 0.51 , and $\mathrm{WBC} 667 / \mu \mathrm{L}$ with lymphocyte dominance $(57 \%)$ but no evidence of malignancy or infection. 
TABLE 1: Lab values during the hospitalization, baseline TSH, free T4, and cortisol levels were within normal range; however, patient presented with elevated TSH, low free T4, and decreased cortisol levels, suggesting immune-mediated hypothyroidism and adrenal insufficiency.

\begin{tabular}{|c|c|c|c|c|c|}
\hline Labs (reference range) & Baseline & Admission & $\begin{array}{l}\text { Day } 7 \text { after } \\
\text { steroid Tx }\end{array}$ & $\begin{array}{l}\text { Day } 21 \text { after } \\
\text { steroid Tx }\end{array}$ & $\begin{array}{c}\text { Day } 35 \text { after } \\
\text { steroid Tx }\end{array}$ \\
\hline $\begin{array}{l}\text { TSH } \\
(0.35-4.00 \mu \mathrm{IU} / \mathrm{mL})\end{array}$ & 3.26 & 6.78 & 8.10 & 5.85 & 2.85 \\
\hline Free T4 (0.7-1.5 ng/dL) & 0.8 & 0.4 & 0.4 & 1.2 & 1.0 \\
\hline ACTH (7-69 pg/mL) & NR & NR & $<5$ & $<5$ & $<5$ \\
\hline $\begin{array}{l}\text { Cortisol at } 8 \mathrm{AM} \\
(4.2-38.4 \mu \mathrm{g} / \mathrm{dL})\end{array}$ & 10.6 & $<1.0$ & $<1.0$ & $<1.0$ & 1.5 \\
\hline AST (5-34 IU/L) & 28 & 30 & 24 & 18 & 21 \\
\hline ALT (0-55 IU/L) & 13 & 14 & 28 & 33 & 19 \\
\hline $\begin{array}{l}\text { Total bilirubin } \\
(0.2-1.2 \mathrm{mg} / \mathrm{dL})\end{array}$ & 1.1 & 1.9 & 0.8 & 0.6 & 0.6 \\
\hline $\begin{array}{l}\text { Troponin I } \\
(0.00-0.02 \mathrm{ng} / \mathrm{mL})\end{array}$ & NR & $<0.02$ & 0.07 & $<0.02$ & NR \\
\hline
\end{tabular}

Brain MRI showed no pathologic changes. TSH, free T4, and morning random cortisol levels after the last cycle of ipilimumab treatment were $3.26 \mu \mathrm{IU} / \mathrm{mL}, 0.8 \mathrm{ng} / \mathrm{dL}$, and $10.6 \mu \mathrm{g} / \mathrm{dL}$, respectively, and rechecked levels on admission showed $6.78 \mu \mathrm{IU} / \mathrm{mL}, 0.4 \mathrm{ng} / \mathrm{dL}$, and $1.0 \mu \mathrm{g} / \mathrm{dL}$, indicating hypothyroidism and adrenal insufficiency (Table 1). Screening colonoscopy prior to ipilimumab treatment had shown normal finding and infectious work-up for the new onset diarrhea including $C$. diff toxin PCR, stool Gram stain, culture, and parasites was all negative. Collectively, these results suggested ipilimumab induced immune-mediated pericarditis, hypothyroidism, adrenal insufficiency, and diarrhea for which high dose intravenous methylprednisolone (125 mg daily) was started. Patient achieved remarkable clinical improvement over the 48 hours, and methylprednisolone was switched to prednisone ( $40 \mathrm{mg}$ daily) and budesonide ( $9 \mathrm{mg}$ daily) on the third day, and they were tapered down over a month. Repeat chest X-ray and CT scan showed resolved pleural and pericardial effusion (Figure 1(d)), and diarrhea improved gradually over the month. Rechecked TSH and random cortisol levels also showed normal range of $2.85 \mu \mathrm{IU} / \mathrm{mL}$ (without thyroid hormone replacement) and $1.5 \mu \mathrm{g} / \mathrm{dL}$, respectively (Table 1 ).

\section{Discussion}

Over the past decades, the incidence of cutaneous melanoma has increased by more than $60 \%$, and $10-15 \%$ of patients present at stage III or IV [1]. Unresectable disease is used to be treated with best supportive care, radiation, or systemic treatments such as dacarbazine and temozolomide; however, the prognosis of metastatic disease is dismal with median survival of less than 12 months [2, 3]. Immune system plays a pivotal role to eradicate cancer cells, making immune modulation a novel therapeutic target. Recognition of various tumor antigens by antigen presenting cells induces cytotoxic $\mathrm{T}$ cell activation via interaction of $\mathrm{T}$ cell receptor with major histocompatibility complex 1 molecule. For the full $\mathrm{T}$ cell activation, additional engagement of costimulatory pathway is required and this is antagonized by CTLA- 4 , an immune check point molecule. CTLA- 4 competes with CD28 to bind CD80 (B7-1) and CD86 (B7-2) and negatively regulates T cell activation and proliferation [4, 5]. Accordingly, CTLA4 knockout mice were shown to develop lymphoproliferative disorder with excessive accumulation of activated T cells and preclinical study with antibodies against CTLA-4 demonstrated tumor cells suppression $[6,7]$.

Ipilimumab is a human monoclonal antibody (IgG1) that blocks the interaction of CTLA-4 with its ligands and recent phase III clinical trials in patients with unresectable metastatic melanoma showed overall survival benefit with ipilimumab treatment compared to gp100 vaccination or dacarbazine monotherapy $[8,9]$. Because antagonizing CTLA4 stimulates $\mathrm{T}$ cell proliferation, ipilimumab treatment is associated with substantial risk of immune mediated adverse reactions and current guideline recommends ipilimumab treatment with careful monitor for these side effects [10]. Previous phase II and III clinical trials showed that grade 3-4 immune related adverse events including enterocolitis, hepatitis, dermatitis, and endocrinopathy can occur in 10$40 \%$ of patients $[5,8,9,11-13]$ and rare complications such as pericarditis, nephritis, pneumonitis, meningitis, uveitis, and hemolytic anemia in less than $1 \%$ of patients who were treated with ipilimumab (Table 2) [14].

The halflife of ipilimumab clearance is 14.7 days [14]; however, immune cell activation and proliferation are slow process [15]. Accordingly, the effect of ipilimumab treatment evolves over months and delayed responses and adverse events (18-20 weeks after treatment) are well known as in this case [15]. Our patient completed the last cycle of ipilimumab treatment 12 weeks prior to admission and he presented with pericarditis and pericardial effusion. Infectious workup including bacterial and viral etiologies was negative. There was no significant history of autoimmune disease and additional examinations for autoimmune disease were all negative. Moreover, pericardial and pleural fluids cytology showed lymphocytes dominance with no evidence of malignancy or infection and pericardial tissue biopsy demonstrated acute inflammation, suggesting ipilimumab induced immune mediated pericarditis and pericardial effusion, most likely. 


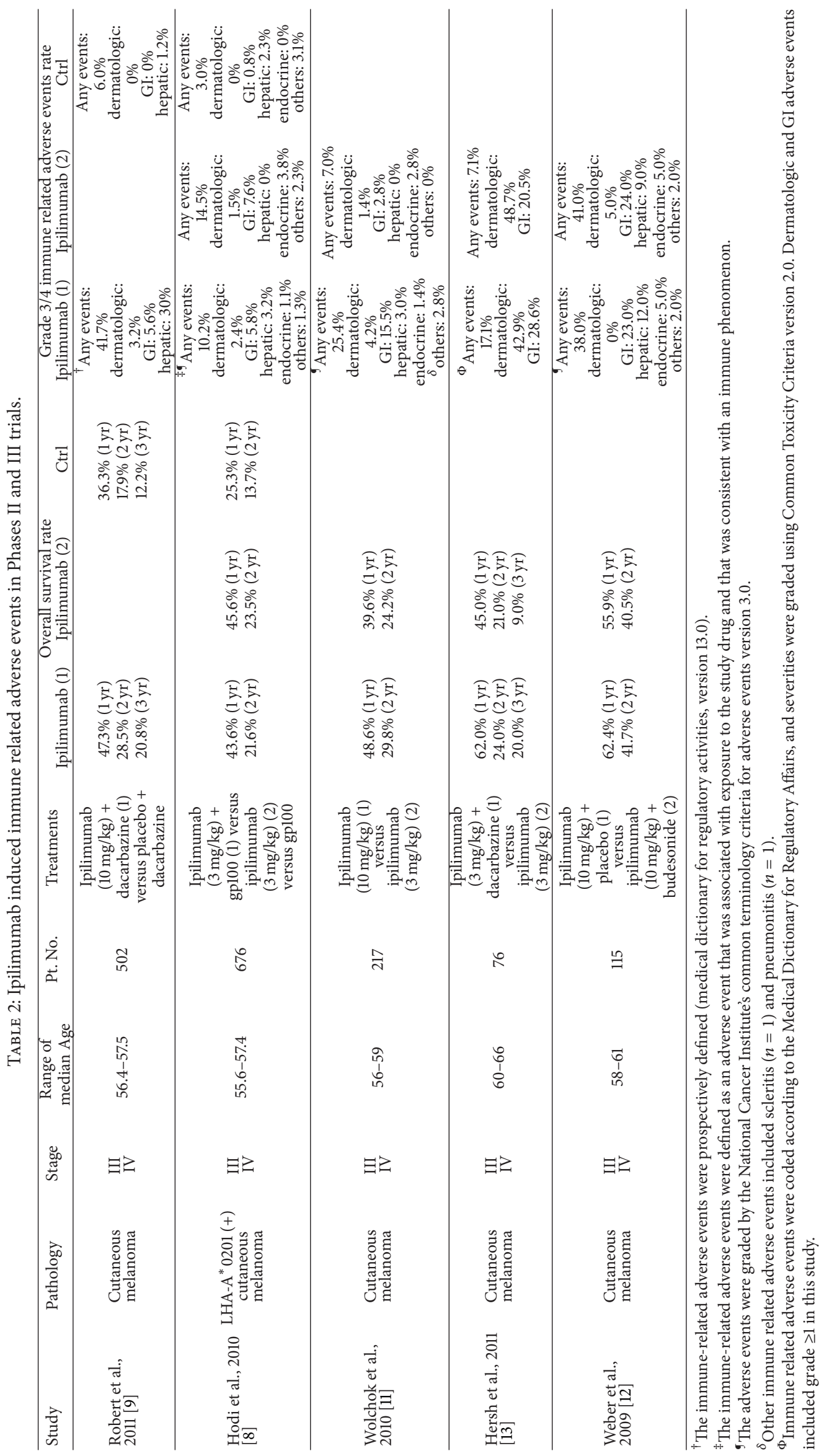


This is supported by associated hypothyroidism, adrenal insufficiency, and diarrhea, all of which showed remarkable improvement with systemic steroid treatment and without hormone replacement.

As shown in phase II and III clinical trials, most of the ipilimumab induced immune related adverse effects are reversible with early recognition and appropriate management. For the severe immune reactions, early administration of high dose systemic corticosteroid is critical and adverse reactions resolve within a median of 2-3 weeks as in our case $[5,8,9,11]$. Collectively, ipilimumab treatment is associated with significant survival benefit and, however, also with life threatening immune mediated adverse effects that require close monitor, early diagnosis, and appropriate management.

\section{Conclusion}

To the best of our knowledge, this is the first case of late onset pericarditis and pericardial effusion associated with ipilimumab treatment in patient with metastatic cutaneous melanoma. Ipilimumab induced immune mediated adverse events could be life threatening as shown in our case, and early diagnosis and intervention with systemic corticosteroid are critical for the better clinical outcome.

\section{Conflict of Interests}

The authors declare that there is no conflict of interests regarding the publication of this paper.

\section{Authors' Contribution}

Seongseok Yun, Nicole D. Vincelette, Dana Hariri, and Iyad Mansour contributed to acquisition, analysis, and interpretation of data. Seongseok Yun, Iyad Mansour, and Dana Hariri were responsible for patient care. Seongseok Yun, Nicole D. Vincelette, Iyad Mansour, and Sara Motamed contributed to drafting the paper.

\section{References}

[1] R. Siegel, J. Ma, Z. Zou, and A. Jemal, "Cancer statistics, 2014," CA: A Cancer Journal for Clinicians, vol. 64, no. 1, pp. 9-29, 2014.

[2] H. Tsao, M. B. Atkins, and A. J. Sober, "Management of cutaneous melanoma," The New England Journal of Medicine, vol. 351, no. 10, pp. 998-1042, 2004.

[3] S. S. Agarwala, "Current systemic therapy for metastatic melanoma," Expert Review of Anticancer Therapy, vol. 9, no. 5, pp. 587-595, 2009.

[4] I. Melero, S. Hervas-Stubbs, M. Glennie, D. M. Pardoll, and L. Chen, "Immunostimulatory monoclonal antibodies for cancer therapy," Nature Reviews Cancer, vol. 7, no. 2, pp. 95-106, 2007.

[5] S. J. O’Day, O. Hamid, and W. J. Urba, "Targeting cytotoxic T-lymphocyte antigen-4 (CTLA-4): a novel strategy for the treatment of melanoma and other malignancies," Cancer, vol. 110, no. 12, pp. 2614-2627, 2007.

[6] P. Waterhouse, J. M. Penninger, E. Timms et al., "Lymphoproliferative disorders with early lethality in mice deficient in Ctla-4," Science, vol. 270, no. 5238, pp. 985-988, 1995.
[7] D. R. Leach, M. F. Krummel, and J. P. Allison, "Enhancement of antitumor immunity by CTLA-4 blockade," Science, vol. 271, no. 5256, pp. 1734-1736, 1996.

[8] F. S. Hodi, S. J. O’Day, D. F. McDermott et al., "Improved survival with ipilimumab in patients with metastatic melanoma," The New England Journal of Medicine, vol. 363, no. 8, pp. 711723, 2010.

[9] C. Robert, L. Thomas, I. Bondarenko et al., "Ipilimumab plus dacarbazine for previously untreated metastatic melanoma," The New England Journal of Medicine, vol. 364, no. 26, pp. 25172526, 2011.

[10] NCCN, NCCN Guideline, Melanoma, Version 2, NCCN, 2015.

[11] J. D. Wolchok, B. Neyns, G. Linette et al., "Ipilimumab monotherapy in patients with pretreated advanced melanoma: a randomised, double-blind, multicentre, phase 2, dose-ranging study," The Lancet Oncology, vol. 11, no. 2, pp. 155-164, 2010.

[12] J. Weber, J. A. Thompson, O. Hamid et al., "A randomized, double-blind, placebo-controlled, phase II study comparing the tolerability and efficacy of ipilimumab administered with or without prophylactic budesonide in patients with unresectable stage III or IV melanoma," Clinical Cancer Research, vol. 15, no. 17, pp. 5591-5598, 2009.

[13] E. M. Hersh, S. J. O’Day, J. Powderly et al., "A phase II multicenter study of ipilimumab with or without dacarbazine in chemotherapy-naïve patients with advanced melanoma," Investigational New Drugs, vol. 29, no. 3, pp. 489-498, 2011.

[14] C. Fellne, "Ipilimumab (yervoy) prolongs survival in advanced melanoma: serious side effects and a hefty price tag may limit its use," $P \&$ T, vol. 37, no. 9, pp. 503-530, 2012.

[15] G. K. Pennock, W. Waterfield, and J. D. Wolchok, "Patient responses to ipilimumab, a novel immunopotentiator for metastatic melanoma: how different are these from conventional treatment responses?" American Journal of Clinical Oncology, vol. 35 , no. 6, pp. 606-611, 2012. 


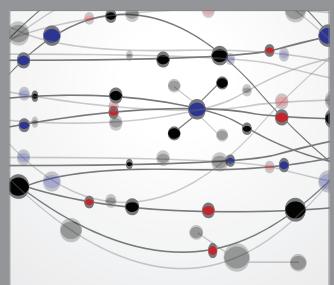

The Scientific World Journal
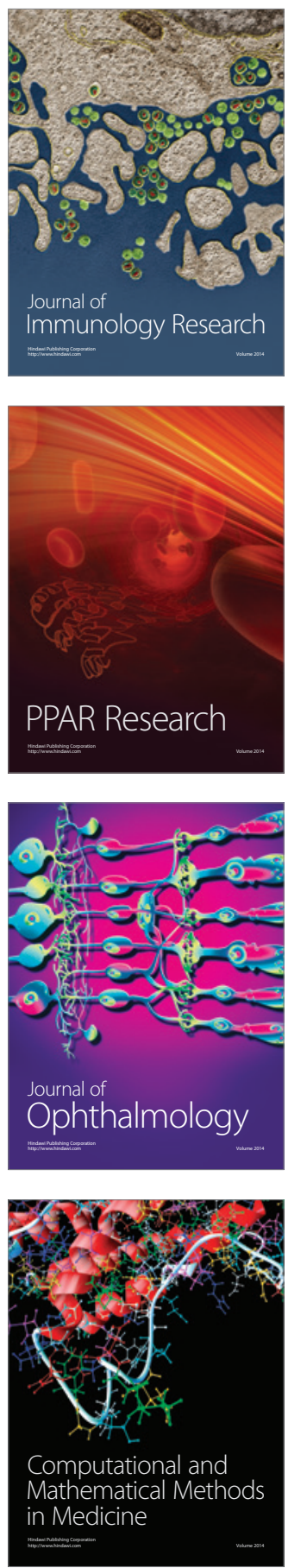

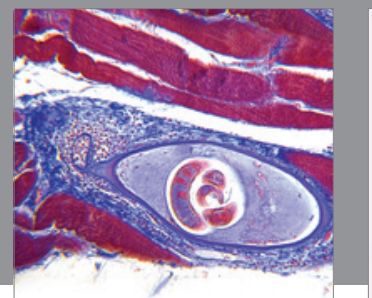

Gastroenterology

Research and Practice
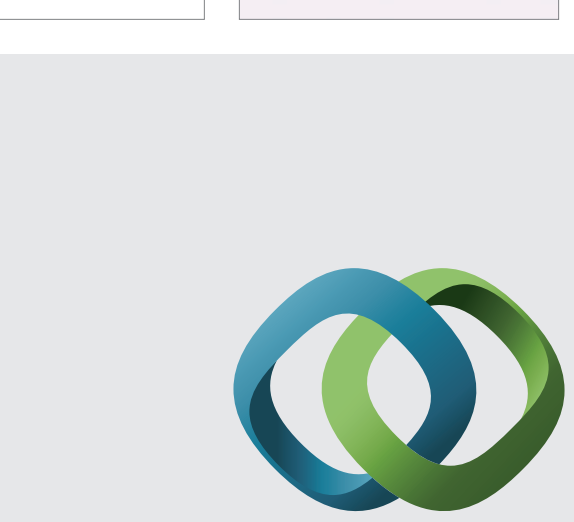

\section{Hindawi}

Submit your manuscripts at

http://www.hindawi.com
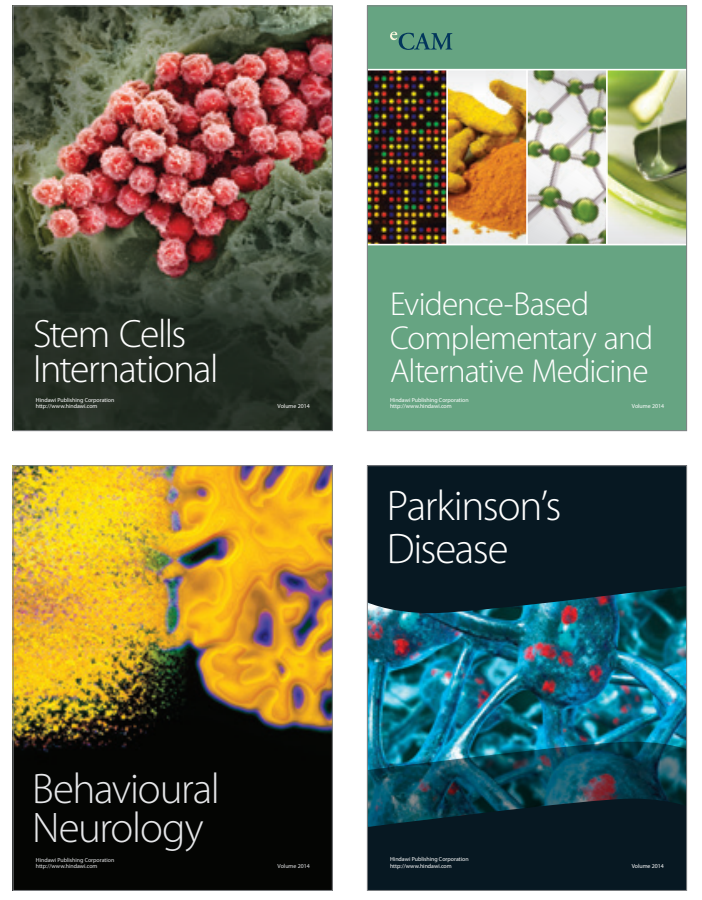
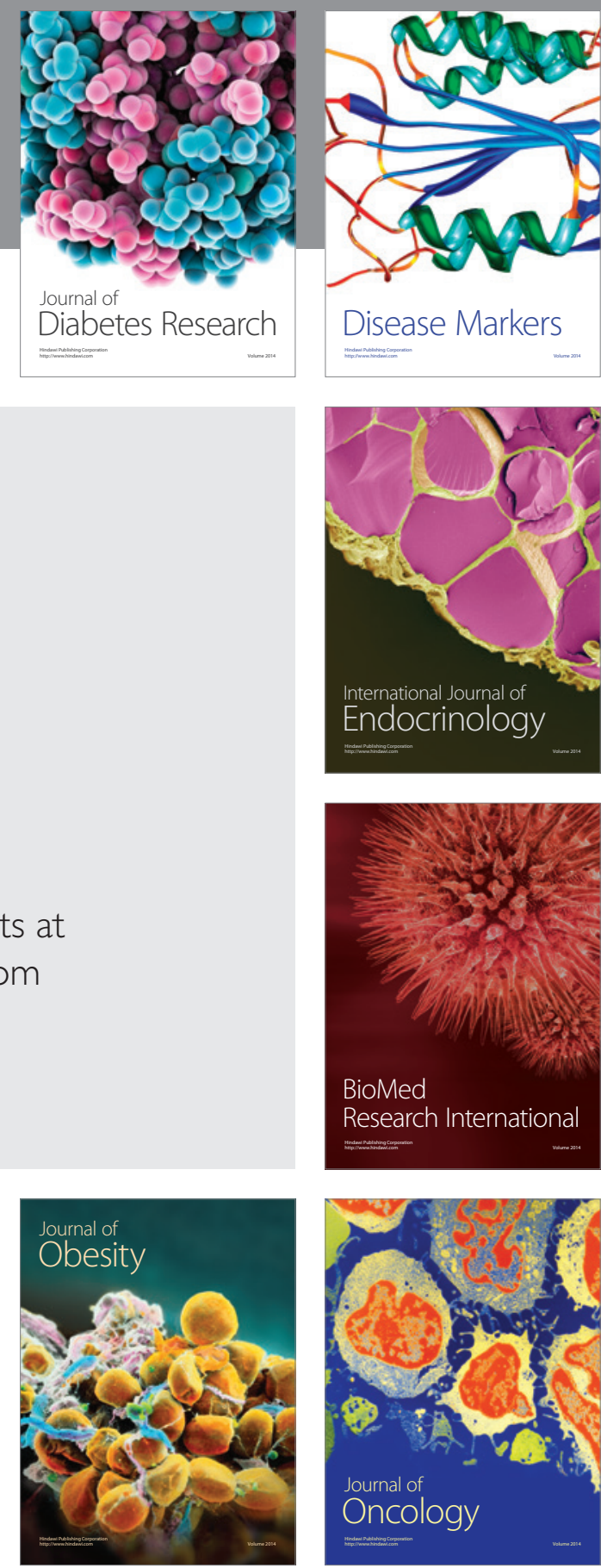

Disease Markers
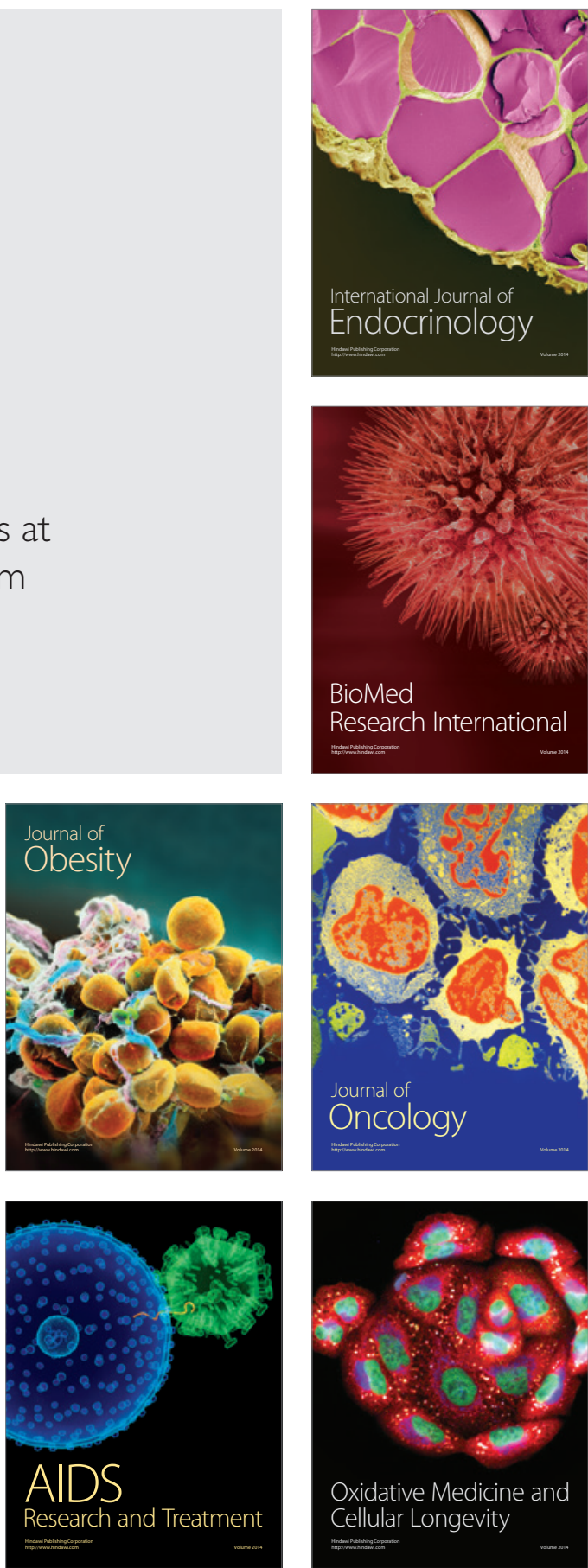